\title{
Determination of Enantiomeric Composition of Substituted Tetrahydroisoquinolines Based on Derivatization with Menthyl Chloroformate
}

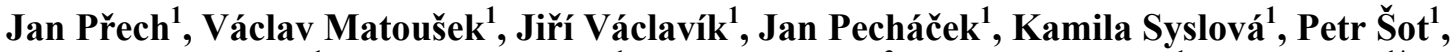 \\ Jakub Januščák ${ }^{1}$, Beáta Vilhanová ${ }^{1}$, Marek Kuzma ${ }^{2}$, Jaromir Toman ${ }^{1}$, Petr Kačer ${ }^{1 *}$ \\ ${ }^{1}$ Department of Organic Technology, Institute of Chemical Technology, Prague, Czech Republic \\ ${ }^{2}$ Laboratory of Molecular Structure Characterization, Institute of Microbiology, \\ Academy of Sciences of the Czech Republic, Prague, Czech Republic \\ Email: *petr.kacer@vscht.cz
}

Received February 3, 2013; revised March 5, 2013; accepted March 17, 2013

\begin{abstract}
A method for the analysis of the optical purity of a series of chiral substituted tetrahydroisoquinolines (THIQs) was developed. The method is based on pre-column derivatization of the analytes with the derivatization reagent (-)$(1 R)$-menthyl chloroformate. The derivatization reaction selectively gives diastereomeric carbamates that are resolvable on an achiral non-polar GC column. The developed technique covers variously substituted THIQs, which differ significantly in volatility, steric and electronic properties. In all cases, the resolution factors $(R)$ exceeded the value of 1.5. The method represents a robust way of analysis of mixtures of THIQs, which are often present in various matrixes such as body fluids, tissues and reaction mixtures.
\end{abstract}

Keywords: Tetrahydroisoquinolines; Enantiomeric Composition; Derivatization; Menthyl Chloroformate; Gas Chromatography

\section{Introduction}

Substituted 1,2,3,4-tetrahydroisoquinolines (THIQs) are substances of both natural and synthetic origin. Their chiral N-heterocyclic scaffold is an integral part of all tetrahydroisoquinoline alkaloids. In a human organism, THIQs affect many physiological and pathological processes. Among the most important endogenously present THIQs are salsolinol (1-methyl-6,7-dihydroxy-THIQ, a catechol isoquinoline) and its derivatives ( $\mathrm{N}$-methyl-salsolinol, norsalsolinol, N-methyl-norsalsolinol), which are all products of reactions of dopamine and aldehydes (e.g. acetaldehyde) catalyzed by the enzyme salsolinol synthase [1] Salsolinol has a physiological function in the organism (regulation of prolactin release, neuronal transmission in sympathetic ganglia, neurotransmission modulation, influence upon the central metabolism and motor activity) but at higher concentrations it causes neurodegenerative diseases such as Parkinson's disease [2]. Higher concentration level of salsolinol has been detected during alcoholism in plasma or urine [3].

Additional examples of important THIQs include noscapine (a benzylisoquinoline alkaloid contained in the

${ }^{*}$ Corresponding author. plants of the Papaveraceae family), which was used for its antitussive (cough-suppressing) effects, $S$-crypto-stylines (isolated from orchids of the genus Cryptostylis), salsoline (isolated e.g. from Corispermum leptopyrum L.) and tubocurarine (known as curare present in the bark of plants Chondrodendron tomentosum). Tubocurarine is a typical non-depolarizing neuromuscular-blocking drug causing the relaxation of skeletal muscles. Since the relaxation is of high importance in surgical procedures, the synthetic derivatives of tubocurarine are commonly used in anaesthesia [4]. Like tubocurarine, these compounds interact with the nicotinic acetylcholine receptors contained in the neuromuscular junction. Due to the optical preference of the receptors, which is very common in general [5], optically pure THIQs (i.e. single enantiomers that exhibit the desired effect) must be administered as the neuromuscular-blocking drugs.

The synthetic derivatives of tubocurarine such as mivacurium chloride and gantacurium chloride can be prepared via the asymmetric transfer hydrogenation (ATH) of prochiral substituted 3,4-dihydroisoquinolines (DHIQs) by using Noyori's chiral half-sandwich catalysts [6-9]. It is important that a universal method for the determination of natural and synthetic THIQ derivatives be devel- 
oped. Such a method would be capable of resolving individual $R$ and $S$ isomers of a wide range of substituted THIQs in various matrixes (reaction mixture, body fluids (plasma, cerebrospinal fluid and urine) and tissues (brain tissue)).

Nowadays, there are several different methods available for the analysis of substituted chiral THIQs. These methods typically employ gas chromatography coupled to mass spectrometry $[10,11]$ and liquid chromatography with UV [12,13], fluorescent [14] or mass-spectrometric detection $[15,16]$. In cases when an achiral GC or LC column is used for the determination of enantiomeric excess, the mixture of enantiomers needs to be reacted with optically pure reagents prior to the analysis $[17,18]$. There are a number of agents available for pre-column derivatization, such as Mosher's acid [19-21], camphanic acid [22], perfluoro-2-propoxypropionylchloride [11] and others. In all cases, resolvable diastereomers are formed in a ratio identical to that of original enantiomers. In the case of substituted THIQs, a GC with a chiral column often cannot be used owing to the high boiling point of these compounds and low stability of the column's stationary phase. For some of 1-methyl and 1-phenyl THIQs, chiral LC methods with polysaccharide [12] or crown ether [13] stationary phase have been developed. Nonetheless, these methods are only applicable to a limited number of analytes due to considerably low resolution of peaks [13].

The determination of the optical purity (i.e. enantiomeric ratio or enantiomeric excess) of chiral THIQs plays an important role both in the pharmaceutical industry and medicinal diagnostics (e.g. Parkinson's disease, predisposition to alcoholism). For that reason, it is highly desirable to develop a method capable of analysis of a wide spectrum of chiral substituted THIQs. In this regard, a suitable method appears to be that which is based on precolumn derivatization (where the reagent reacts with the nitrogen atom in the heterocycle) in a combination with high performance gas chromatography on an achiral column coupled with mass-spectrometric detection.

\section{Experimental}

\subsection{Chemicals}

The following chemicals and reagents were of comercial origin: $(-)-(1 R)$-menthyl chloroformate $(99 \%$; ee $=99.9 \%)$; triethylamine (99.5\%) (Sigma-Aldrich, Germany); sodium borohydride (97\%) (CMS Chemicals, Slovakia); acetonitrile (99.5\%), methanol (99.5\%); diethyl ether (99\%), anhydrous potassium carbonate (p.a.), sodium hydroxide (98\%) (Penta, Czech Republic).

Substituted chiral THIQs 1-methyl-THIQ (1); 6-methoxy-1-methyl-THIQ (2); 7-methoxy-1-methyl-THIQ (3); 6,7-dimethoxy-1-methyl-THIQ (4); 1-phenyl-THIQ (5); 1-(4-methylphenyl)-THIQ (6); 1-(4-methoxyphenyl)-THIQ
(7) and 1-[4-(trifluoromethyl)phenyl]-THIQ (8) (Figure 1) were prepared in both racemic and enantioenriched form, the former by non-stereoselective sodium tetrahydridoborate reduction, the latter by ATH of corresponding DHIQs according to Noyori protocol [6]. Substituted DHIQs were synthesized by Bischler-Napieralski cyclodehydration from corresponding phenethylamides according to our previously reported procedure [23].

\subsection{Instrumentation}

GC analysis was carried out on a Varian CP 3800 equipped with a flame-ionization detector (Varian FID 11), Varian 1177 injector, Varian VF-1 capillary column (60 $\mathrm{m} \times 0.25 \mathrm{~mm} \times 0.25 \mu \mathrm{m}$ ) and Varian Combi/Liquid PAL system autosampler. The oven temperature program is described in Table 1. The flow of carrier gas (nitrogen, $99.99 \%$ ) was $0.5 \mathrm{~mL} / \mathrm{min}$, the temperature of injector was $300^{\circ} \mathrm{C}$, the split ratio was 1:25 and the injection volume was $1 \mu \mathrm{L}$. The temperature of detector was $250^{\circ} \mathrm{C}$.

Structure determination of derivatized THIQs was performed using a GC-MS system consisting of Varian CP 3800 coupled to a Saturn 2000 mass spectrometer equipped with electron impact ionization and an ion trap.

The system was equipped with VF-1ms capillary column $(60 \mathrm{~m} \times 0.25 \mathrm{~mm} \times 0.25 \mu \mathrm{m})$. The carrier gas was helium (99.9999\%). The GC method was identical to the one described above for $\mathrm{GC}$ analyses. The temperatures of transfer line and ion trap were $180^{\circ} \mathrm{C}$ and $150^{\circ} \mathrm{C}$, respectively. Standard electron impact conditions $(70 \mathrm{eV})$ were used. The solvent delay was set to 10 minutes.

\subsection{Derivatization Procedure}

Racemic or enantiomerically enriched THIQ (typically 3 $\mathrm{mg} ; 0.01$ - $0.02 \mathrm{mmol})$ was dissolved in acetonitrile (1 $\mathrm{mL})$ and triethylamine (TEA) $(20 \mu \mathrm{L}, 0.14 \mathrm{mmol})$ was added followed by $(-)-(1 R)$-menthyl chloroformate $(10$ $\mu \mathrm{L}, 0.03 \mathrm{mmol})$. The mixture was reacted for 10 minutes at room temperature (Figure 2) and directly analyzed on GC.

For samples containing $3-6 \mathrm{mg}$ of the THIQs, the amounts of the reagents were doubled.<smiles>[R]c1cc2c(cc1[R])[C@H](C)NCC2</smiles>

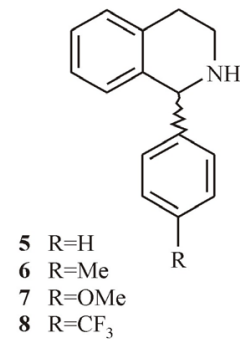

Figure 1. Structures of the chiral tetrahydroisoquinolines used. 


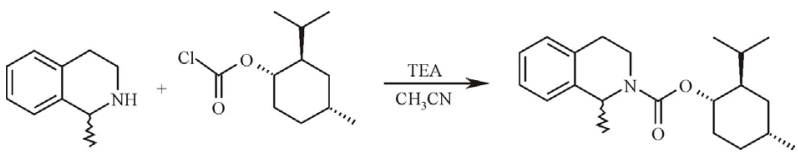

Figure 2. Derivatization reaction of 1-methyl-THIQ (1).

Table 1. Temperature program of the column.

\begin{tabular}{ccc}
\hline Temperature $\left({ }^{\circ} \mathrm{C}\right)$ & Rate $\left({ }^{\circ} \mathrm{C} / \mathrm{min}\right)$ & Hold time $(\mathrm{min})$ \\
\hline 80 & & 5 \\
200 & 10 & 20 \\
245 & 10 & 55 \\
265 & 20 & 30 \\
\hline
\end{tabular}

\subsection{Calculations}

Separation parameters in the optimization of the chromatographic conditions and enantiomeric excess were defined as follows:

$$
\begin{gathered}
R=\frac{t_{2}-t_{1}}{0.5 \times\left(w_{1}+w_{2}\right)} \\
\alpha=\frac{t_{2}}{t_{1}} \\
e e=\frac{c_{\text {maj }}-c_{\text {min }}}{c_{\text {maj }}+c_{\text {min }}}
\end{gathered}
$$

Symbols $R, \alpha$ and ee represent the resolution factor, selectivity factor and enantiomeric excess, respectively. Symbols $t, w$ and $c$ are retention time, peak width at the baseline and concentration of the diastereomer. The suffixes 1 and 2 correspond to the first and second detected diastereomer. The suffixes maj and min refer to the major or minor diastereomer in a non-racemic mixture. As the concentrations of the enantiomers were determined always in one analysis, they stood as internal standards to each other.

\subsection{Method Performance}

The following parameters were determined during the method validation: limit of detection (LOD), limit of quantification (LOQ), linear range, accuracy and precision.

The calibration lines were evaluated under optimal chromatographic conditions by injection of 5 standard solutions of derivatized racemic THIQs and peak area measurement. Calibration equations, correlation coefficients $(R 2)$ and linear ranges were determined.

Detection and quantification limits were estimated by analyzing racemic standard solutions at low concentrations. LODs and LOQs were determined as the lowest concentration of the analyte that produced chromatographic peak at a signal to noise ratio of 3 and 10 , respectively.
The accuracy and precision of the method was established through the measurements of enantiomer retention times and peak areas using standard solutions of 1 $\mathrm{mg} / \mathrm{mL}$. Precision is expressed as the repeatability (RSD, $\%$ ) of four consecutive injections. For inter-day precision and carbamate stability, derivatized samples were analyzed daily over a 3 -day period.

\section{Results and Discussion}

The chromatogram of derivatized THIQs (Figure 3) contained no signals of non-derivatized amines, indicating $100 \%$ conversion to corresponding carbamates. The structure of the carbamates was confirmed by GC-MS analysis (Figure 4). To the best of our knowledge, the spectra of these compounds have not been published before.

The ee value for compound 4 agreed with the one reported previously [6] by using chiral HPLC column. Compounds 1, 4 and 5 were also analyzed using a chiral HPLC method described in [24], obtaining ee values identical to those obtained by GC analysis of derivatized THIQs.

Retention times of the carbamates are summarized in Table 2, together with their resolution and separation factors determined at the concentrations of THIQs $c=$ $0.5 \mathrm{mg} / \mathrm{mL}$. The peaks within $10-30 \mathrm{~min}$ represent the unreacted excess of $(-)-(1 R)$-menthyl chloroformate $(t=$ $27.12 \mathrm{~min})$ and products of its thermal decomposition during evaporation of the sample (mainly 2-(prop-2-yl)5 -methylcyclohexanol, according to the GC-MS analysis; $t=14.62 \mathrm{~min})$.

\begin{tabular}{|c|c|c|c|}
\hline Substance & r.t. ${ }^{\text {a }}(\min )$ & $\begin{array}{c}\text { Resolution } \\
(R)\end{array}$ & $\begin{array}{c}\text { Separation } \\
(\alpha)\end{array}$ \\
\hline$(R)-1 \mathrm{Me}-\mathrm{THIQ}$ & 54.19 & \multirow{2}{*}{2.19} & \multirow{2}{*}{1.010} \\
\hline$(S)-1 \mathrm{Me}-\mathrm{THIQ}$ & 54.74 & & \\
\hline$(R)-7 \mathrm{MeO}-1 \mathrm{Me}-\mathrm{THIQ}$ & 68.70 & \multirow{2}{*}{2.71} & \multirow{2}{*}{1.015} \\
\hline$(S)-7 \mathrm{MeO}-1 \mathrm{Me}-\mathrm{THIQ}$ & 69.76 & & \\
\hline$(R)-6 \mathrm{MeO}-1 \mathrm{Me}-\mathrm{THIQ}$ & 70.25 & \multirow{2}{*}{2.72} & \multirow{2}{*}{1.016} \\
\hline$(S)-6 \mathrm{MeO}-1 \mathrm{Me}-\mathrm{THIQ}$ & 71.40 & & \\
\hline$(R)-1-\left(4-\mathrm{CF}_{3} \mathrm{Ph}\right)-\mathrm{THIQ}$ & 82.22 & \multirow{2}{*}{2.26} & \multirow{2}{*}{1.011} \\
\hline$(S)-1-\left(4-\mathrm{CF}_{3} \mathrm{Ph}\right)-\mathrm{THIQ}$ & 83.10 & & \\
\hline (R)-6,7-diMeO-1Me-THIQ & 86.72 & \multirow{2}{*}{3.42} & \multirow{2}{*}{1.016} \\
\hline$(S)-6,7-d i M e O-1 M e-T H I Q$ & 88.14 & & \\
\hline (R)-1Ph-THIQ & 91.64 & \multirow{2}{*}{2.15} & \multirow{2}{*}{1.012} \\
\hline$(S)-1 \mathrm{Ph}-\mathrm{THIQ}$ & 92.71 & & \\
\hline$(R)-1-(4-\mathrm{MePh})-\mathrm{THIQ}$ & 100.92 & \multirow{2}{*}{1.68} & \multirow{2}{*}{1.008} \\
\hline$(S)-1-(4-\mathrm{MePh})-\mathrm{THIQ}$ & 101.71 & & \\
\hline (R)-1-(4-MeOPh)-THIQ & 116.31 & \multirow{2}{*}{1.88} & \multirow{2}{*}{1.009} \\
\hline$(S)-1-(4-\mathrm{MeOPh})-\mathrm{THIQ}$ & 117.31 & & \\
\hline
\end{tabular}

Table 2. Retention times, resolution and separation factors of the derivatized THIQs.

${ }^{\mathrm{a}}$ Retention times of the corresponding carbamates. 


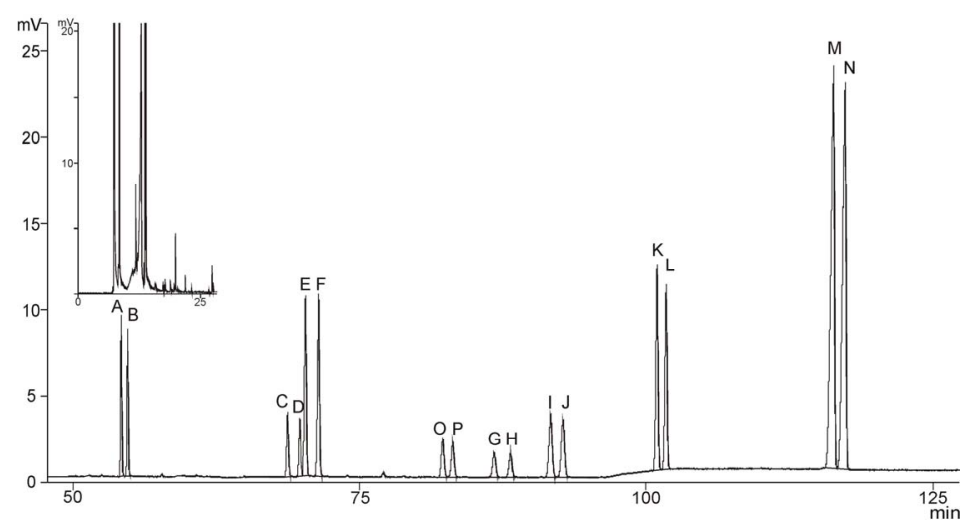

Figure 3. Chromatogram of a mixture of derivatized tetrahydroisoquinolines. EI-MS spectra for peaks A)-P) are given in Figure 4.
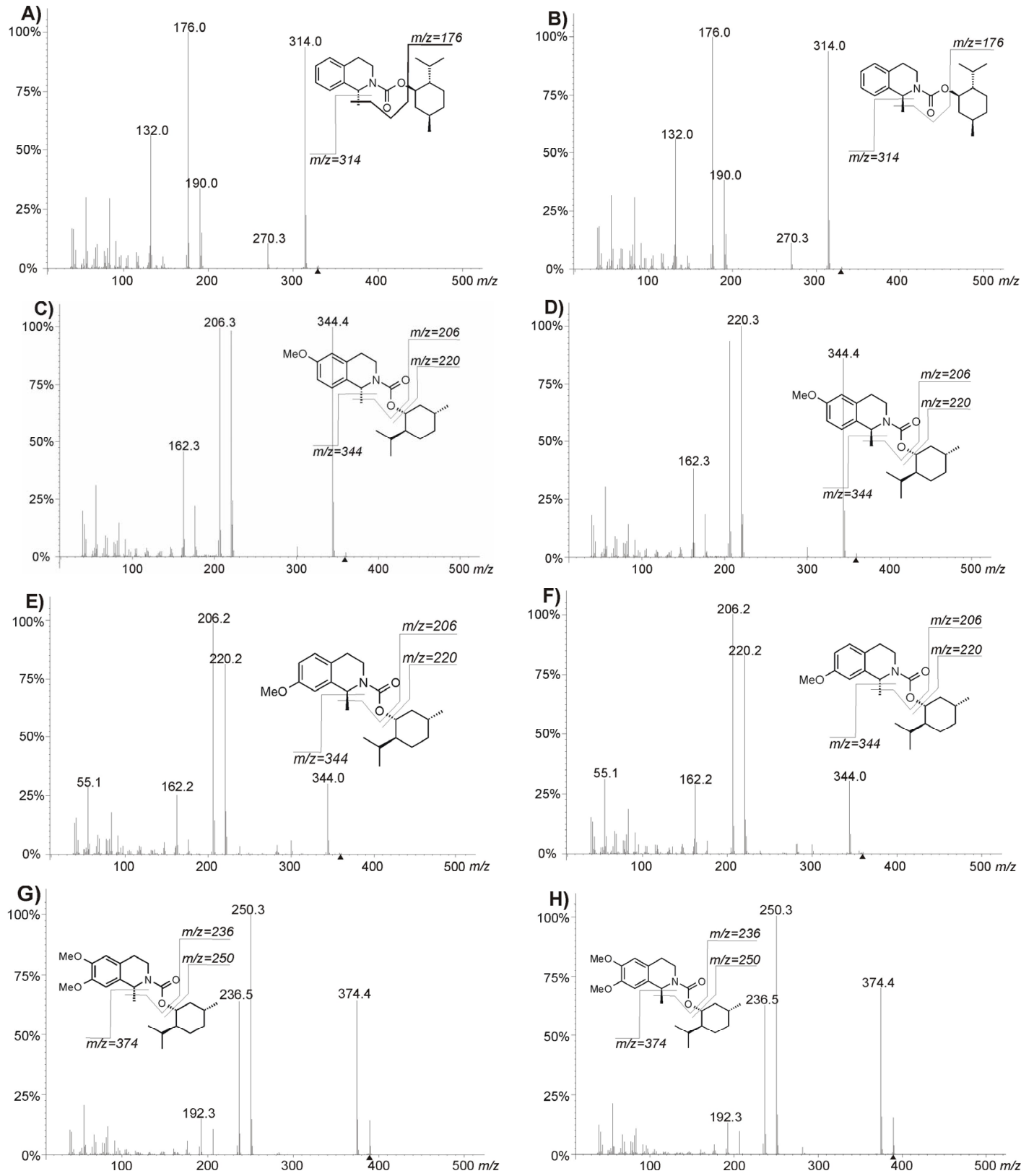

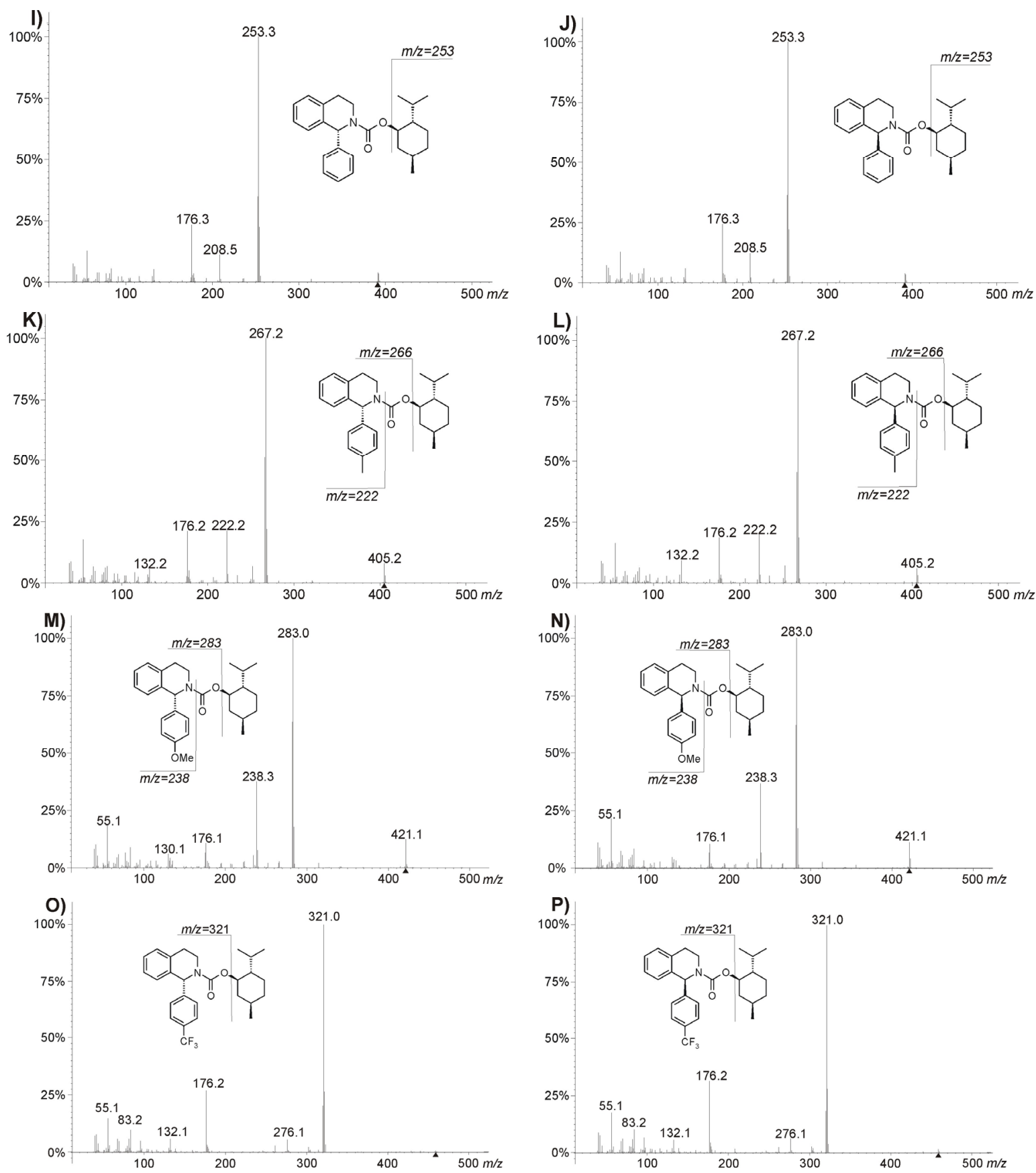

Figure 4. EI-MS spectra of diastereomeric carbamates corresponding to both enantiomers of 1-methyl-THIQ A), B), 6methoxy-1-methyl-THIQ C), D) and 7-methoxy-1-methyl-THIQ E), F) 6,7-dimethoxy-1-methyl-THIQ G), H), 1-phenylTHIQ I), J), 1-(4-methylphenyl)-THIQ K), L), 1-(4-methoxyphenyl-THIQ M), N) and 1-(4-trifluoromethylphenyl)-THIQ O), P).

\subsection{Robustness of the Derivatization Method}

No remarkable loss of response or change in the ratio of diastereomeric peaks was observed during a 5-day period. Experiments using less than 1 molar eq of $(-)-(1 R)$-menthyl chloroformate for the derivatization of racemate did not confirm any preferential derivatization of particular enantiomer (i.e. chiral discrimination). It was possible to derivatize THIQs even in large excess of competing nucleophiles (20 molar eq of ethanol or 20 molar eq of morpholine) indicating robustness of this methodology. High separation efficiency of the GC column even allowed us to determine the enantiomeric composition of two regioisomeric THIQs 2 and 3 present simultaneously in a mixture (Figure 3). Resolution factor of the second diastereomeric peak of 3 and the first diastereomeric peak 
of 2 was $R=1.22$.

\subsection{Main EI Fragmentation Routes}

The mass spectra of diastereomeric carbamates are presented in Figure 4. The molecular ion peaks were either missing (carbamates corresponding to 1, 2, 3, 4 and 8), or were present with weak intensity (carbamates corresponding to 5,6 and 7). The main features of all spectra were ions of $m / z=\mathrm{M}-138$, being usually the base peak (BP), or having intensities similar to the BP. Those signals represented the neutral loss of $p$-isopropylmethyl-cyclohexene - a fragment of the menthyl moiety. The heaviest ions for 1-methyl substituted THIQ carbamates were signals $m / z=\mathrm{M}-15$, representing the loss of a methyl radical. The methyl substituent was lost from position 1 in the THIQ skeleton because similar signals in the spectra of 1-phenyl THIQ carbamates were missing. The heaviest ions in the spectra of 1-phenyl THIQ carbamates were the molecular ions with low intensity and mentioned $\mathrm{m} / \mathrm{z}$ $=\mathrm{M}-138$ radical. The fragmentation patterns for both 1methyl-THIQ carbamates and 1-phenyl-THIQ carbamates are summarized in Figure 5.

The THIQ skeleton appears to be the most stable fragment of the molecule as evidenced by lighter signals in the spectra, which represents this part of the molecule losing other substituents. For instance, $m / z=\mathrm{M}-183$ represents the loss of entire menthyl-formate fragment (carbamates corresponding to $5,6,7$ and 8 ) and $m / z=176$ (carbamates corresponding to $1,5,6,7$ and 8 ) represents the loss of $p$-isopropyl(methyl)cyclohexene together with the substituent in position 1 of THIQ.

The lightest considerable signals were $\mathrm{m} / \mathrm{z}=132$ (carbamates corresponding to $1,5,6,7$ and 8$), m / z=162$ (carbamates corresponding to 2 and 4), and $m / z=192$ (carbamate corresponding to 4), which belong to discrete THIQ skeleton bearing only substituents in positions 6 and 7, if applicable (molecules 2 - 4). Signals below $\mathrm{m} / \mathrm{z}$ $=100$ represent a mixture of small fragments of the molecule and have generally low intensity, e.g. fragment ions of the THIQ skeleton could be found $(\mathrm{m} / \mathrm{z}=91,83$, 55 and 39 ).

\subsection{Method Validation}

The method performance was evaluated based on the following analytical parameters: LOQ, LOD, linear range accuracy and precision. LOQ and LOD values were estimated as described in Section 2.5 by injection of diluted samples with defined concentration of starting THIQs. The diluted samples were derivatized with standard amounts of reagents before the analysis.

The determination of the linearity of the instrumental response was based on peak area. Calibration standards of racemic THIQs were prepared in acetonitrile at concentrations ranging from $10 \mu \mathrm{g} / \mathrm{mL}$ (average LOQ for the THIQs, see Table 3) to $5 \mathrm{mg} / \mathrm{mL}$. Linear regression ana-

Table 3. Validation parameters of the method.

\begin{tabular}{|c|c|c|c|c|c|c|c|}
\hline Substance & $\begin{array}{l}\text { LOD } \\
(\mu \mathrm{g} / \mathrm{ml})\end{array}$ & $\begin{array}{l}\text { LOQ } \\
(\mu \mathrm{g} / \mathrm{ml})\end{array}$ & $\begin{array}{l}\text { Linear range } \\
(\mu \mathrm{g} / \mathrm{ml})\end{array}$ & $\begin{array}{l}\text { Regression eq. }{ }^{\mathrm{a}} \\
(n=5)\end{array}$ & $\begin{array}{l}\text { Correlation coeff. } \\
\left(R^{2}\right)\end{array}$ & $\begin{array}{l}\text { Repeatability } \\
(\%)\end{array}$ & $\begin{array}{l}\text { Inter-day stability }{ }^{\mathrm{d}} \\
(\%)\end{array}$ \\
\hline$(R)-1 \mathrm{Me}-\mathrm{THIQ}$ & 5 & 20 & $10-10,000$ & $y=0.54272+420.31 x$ & 0.9998 & 2.65 & 0.28 \\
\hline$(S)-1 \mathrm{Me}-\mathrm{THIQ}$ & 5 & 20 & $10-10,000$ & $y=2.4942+407.84 x$ & 0.9998 & 1.64 & 0.39 \\
\hline (R)-7MeO-1Me-THIQ & 2 & 5 & $10-10,000$ & $y=0.10995+339.23 x$ & 0.9998 & 3.12 & 0.54 \\
\hline (S)-7MeO-1Me-THIQ & 2 & 5 & $10-10,000$ & $y=-0.2423+341.43 x$ & 0.9997 & 3.65 & 0.65 \\
\hline (R)-6MeO-1Me-THIQ & 2 & 5 & $10-10,000$ & $y=-0.49441+1963.15 x$ & 0.9999 & 2.05 & 0.58 \\
\hline (S)-6MeO-1Me-THIQ & 2 & 5 & $10-10,000$ & $y=-0.86383+1963.70 x$ & 0.9999 & 3.31 & 0.46 \\
\hline$(R)-1-\left(4-\mathrm{CF}_{3} \mathrm{Ph}\right)-\mathrm{THIQ}$ & 6 & 20 & $20-10,000$ & $y=7.899+265.91 x$ & 0.9932 & 3.20 & 1.34 \\
\hline$(S)-1-\left(4-\mathrm{CF}_{3} \mathrm{Ph}\right)-\mathrm{THIQ}$ & 6 & 20 & $20-10,000$ & $y=10.77+193.35 x$ & 0.9926 & 1.54 & 2.06 \\
\hline (R)-6,7-diMeO-1Me-THIQ & 3 & 10 & $10-10,000$ & $y=0.04633+190.43 x$ & 0.9992 & 2.88 & 0.03 \\
\hline (S)-6,7-diMeO-1Me-THIQ & 3 & 10 & $10-10,000$ & $y=-0.2500+185.78 x$ & 0.9993 & 3.40 & 1.13 \\
\hline$(R)-1 \mathrm{Ph}-\mathrm{THIQ}$ & 2 & 10 & $10-10,000$ & $y=2.258+455.20 x$ & 0.9965 & 1.95 & 0.62 \\
\hline$(S)-1 \mathrm{Ph}-\mathrm{THIQ}$ & 2 & 10 & $10-10,000$ & $y=3.511+400.13 x$ & 0.9902 & 2.64 & 0.75 \\
\hline (R)-1-(4-MePh)-THIQ & 3 & 10 & $10-10,000$ & $y=0.169+315.10 x$ & 0.9984 & 2.30 & 1.81 \\
\hline (S)-1-(4-MePh)-THIQ & 3 & 10 & $10-10,000$ & $y=0.845+278.98 x$ & 0.9966 & 1.64 & 2.48 \\
\hline (R)-1-(4-MeOPh)-THIQ & 3 & 10 & $20-10,000$ & $y=-7.875+263.8 x$ & 0.9999 & 2.48 & 0.88 \\
\hline (S)-1-(4-MeOPh)-THIQ & 3 & 10 & $20-10,000$ & $y=-8.120+257.8 x$ & 0.9999 & 1.86 & 1.03 \\
\hline
\end{tabular}

${ }^{\mathrm{a}}$ Calibration curve as the response of the detector $(y)$ dependent on the concentration $(x[\mathrm{mg} / \mathrm{ml}])$ of the sample before derivatization. ${ }^{\mathrm{b}}$ Correlation coefficient of the calibration curve. ${ }^{\mathrm{c}}$ Repeatability determined as relative standard deviation $n=4 ; c=100 \mu \mathrm{g} / \mathrm{mL}$. ${ }^{\mathrm{d}}$ Inter-day stability determined as relative standard deviation $n=3 ; c=20 \mu \mathrm{g} / \mathrm{mL}$. 


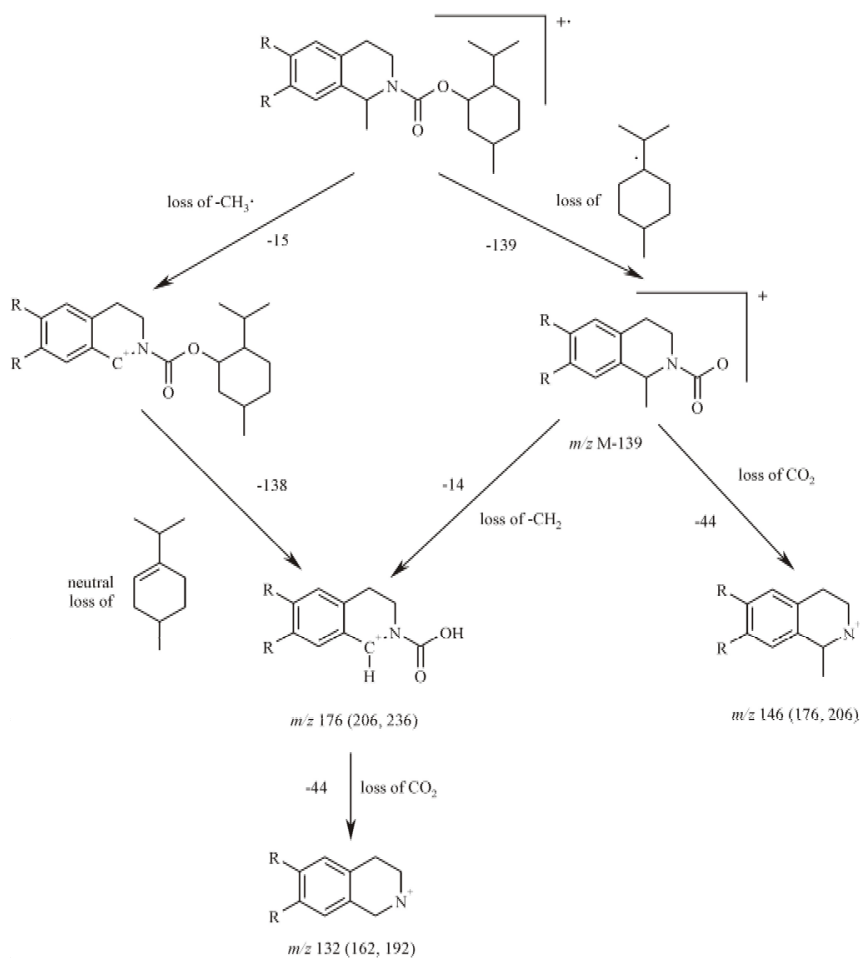

(a)

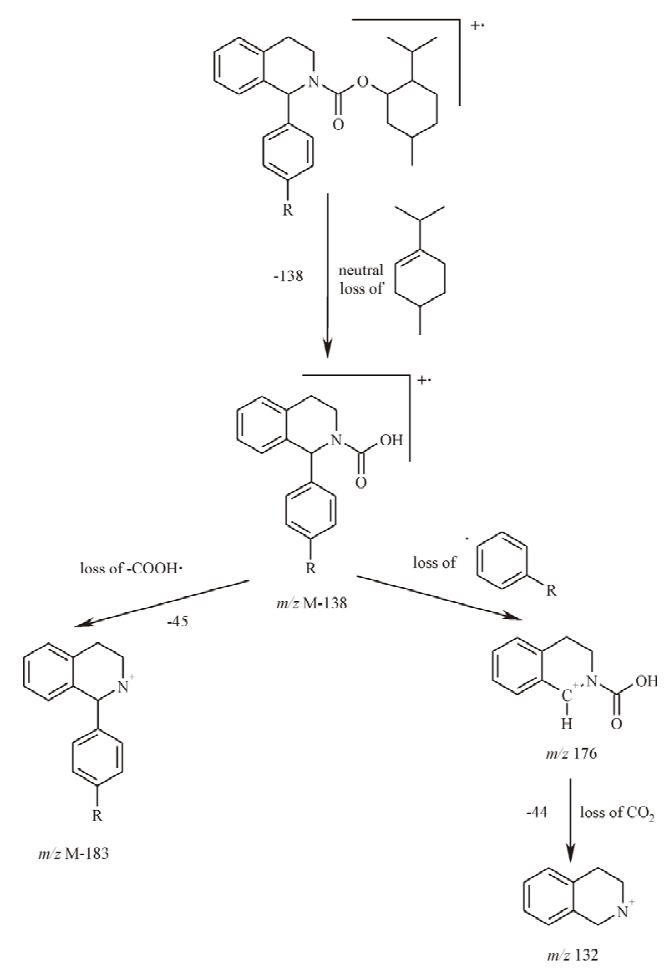

(b)

Figure 5. Proposed fragmentation pattern for derivatized (a) 1-methyl and (b) 1-phenyl substituted tetrahydroisoquinolines.

lysis was applied to plots of peak area vs concentration using the least-square method. Excellent linearity was observed at concentrations within the tested interval. The correlation coefficients $\left(r^{2}\right)$ were higher than 0.99 for all the compounds.

\subsection{Example of Application}

The ATH of cyclic imines has become a well-established route toward the production of optically enriched tertiary amines [25] and therefore a method for the determination of $e e$ is highly desirable in this field. We carried out a kinetic experiment consisting in the ATH of a prochiral DHIQ to a THIQ catalyzed by $\operatorname{RuCl}\left(\eta^{6}-p\right.$-cymene) $[(S, S)$ TsDPEN] (TsDPEN $=N$ - $p$-tosyl-1,2-diphenylethylene-1, 2-diamine) [6]. The developed method was successfully applied in the determination of the product's $e e$.

Figure 6 represents a typical conversion and ee profile of the ATH of 1-methyl-3,4-dihydroisoquinoline (1-MeDHIQ) to 1 in acetonitrile at $30^{\circ} \mathrm{C}$ and $1-\mathrm{Me}-\mathrm{DHIQ} /$ catalyst molar ratio $(\mathrm{S} / \mathrm{C})$ of 100 . The azeotropic mixture of formic acid and triethylamine $(5: 2 \mathrm{~mol} / \mathrm{mol})$ was used as a hydrogen source and the initial concentration of 1$\mathrm{Me}$-DHIQ was $0.344 \mathrm{~mol} / \mathrm{L}$. The $e e(81.8 \% \pm 0.34 \%)$ remained constant during the reaction.

\section{Conclusion}

A rapid and simple derivatization method was developed

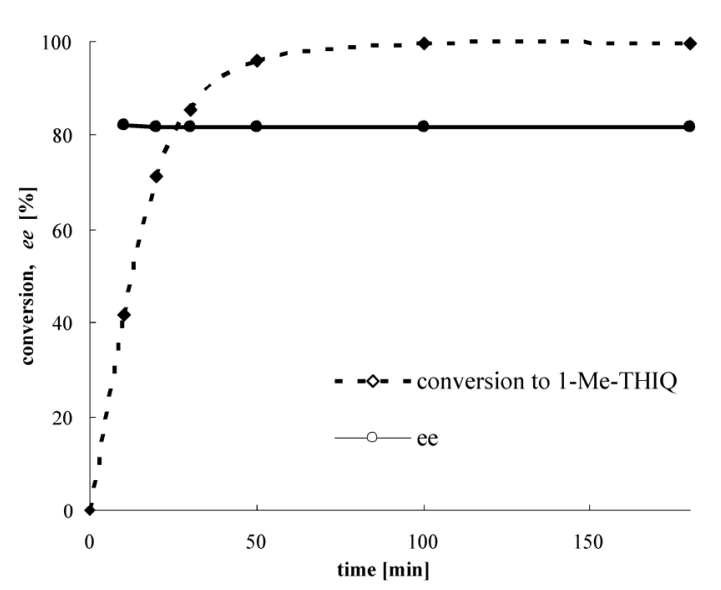

Figure 6. Development of conversion and $e e$ in the course of the ATH of 1-methyl-3,4-dihydroisoquinoline to 1-methyl THIQ (1).

for the determination of enantiomeric composition of variously substituted THIQs. The method proved robust since all analytes were easily resolvable by using a GC with an achiral column after pre-column derivatization. Optically pure (-)-(1R)-menthyl chloroformate, was shown to be a cheap and readily accessible chiral derivatization reagent. By reacting with the nitrogen atom of the THIQ scaffold at room temperature, diastereomeric carbamates were formed, which were resolvable on an ordinary nonpolarachiral GC column. The method represents a facile 
way of determination of enantiomeric purity of a variety of THIQs. Its application lies in the area of pharmaceutical industry and medicinal diagnostics since such compounds are present in the organism, and a number of commonly-used drugs are based on the THIQ structure.

\section{Acknowledgements}

The authors would like to acknowledge the Grant Agency of the Czech Republic for funding this work through research grants GACR 104/09/1497 and P106/12/1276.

\section{REFERENCES}

[1] W. Maruyama, G. Sobue, K. Matsubara, Y. Hashizume, P. Dostert and M. Naoi, "A Dopaminergic Neurotoxin, 1 $(R)$, 2(N)-Dimethyl-6,7-dihydroxy-1,2,3,4-tetrahydroisoquinol ine, $N$-Methyl $(R)$ salsolinol, and Its Oxidation Product, 1 , 2(N)-Dimethyl-6,7-dihydroxyisoquinolinium Ion, Accumulate in the Nigro-Striatal System of the Human Brain," Neuroscience Letters, Vol. 223, No. 1, 1997, pp. 61-64. doi:10.1016/S0304-3940(97)13389-4

[2] W. Zhu, D. Wang, J. Zheng, A. Yarui, Q. Wang, W. Zhang, L. Jin, H. Gao and L. Lin, "Effect of $(R)$-Salsolinol and $N$-Methyl- $(R)$-Salsolinol on the Balance Impairment between Dopamine and Acetylcholine in Rat Brain: Involvement in Pathogenesis of Parkinson Disease," Clinical Chemistry, Vol. 54, No. 4, 2008, pp. 705-712. doi:10.1373/clinchem.2007.097725

[3] H. Haber, A. Winkler, I. Putscher, P. Henklein, I. Baeger, M. Georgi and M. F. Melzig, "Plasma and Urine Salsolinol in Humans: Effect of Acute Ethanol Intake on the Enantiomeric Composition of Salsolinol," Alcoholism: Clinical and Experimental Research, Vol. 20, No. 1, 1996, pp. 87-92. doi:10.1111/j.1530-0277.1996.tb01049.x

[4] H. Ihmsen, J. Schmidt, H. Schwilden, H. J. Schmitt and T. Muenster, "Influence of Disease Progression on the Neuromuscular Blocking Effect of Mivacurium in Children and Adolescents with Duchenne Muscular Dystrophy," Anesthesiology, Vol. 110, No. 5, 2009, pp. 1016-1019. doi:10.1097/ALN.0b013e31819daf31

[5] J. Caldwell, "The Importance of Stereochemistry in Drug Action and Disposition," Journal of Clinical Pharmacology, Vol. 32, No. 10, 1992, pp. 925-929.

[6] N. Uematsu, A. Fujii, S. Hashiguchi, T. Ikariya and R. Noyori, "Asymmetric Transfer Hydrogenation of Imines," Journal of American Chemical Society, Vol. 118, No. 20, 1996, pp. 4916-4917. doi:10.1021/ja960364k

[7] V. Samano, J. A. Ray, J. B. Thompson, R. A. Mook, D. K. Jung, C. S. Koble, M. T. Martin, E. C. Bigham, C. S. Regitz, P. L. Feldman and E. E. Boros, "Synthesis of Ultra-Short-Acting Neuromuscular Blocker GW 0430: A Remarkably Stereo- and Regioselective Synthesis of Tetrahydroisoquinolinium Chlorofumarates," Organic Letters, Vol. 1, No. 12, 1999, pp. 1993-1996. doi:10.1021/o19911573

[8] M. Ružič, A. Pečavar, D. Prudič, D. Kralj, C. Scriban and A. Zanotti-Gerosa, "The Development of an Asymmetric Hydrogenation Process for the Preparation of Solifena- cin," Organic Process Research \& Development, Vol. 16 No. 7, 2012, pp. 1293-1300. doi:10.1021/op3000543

[9] B. Vilhanová, V. Matoušek, J. Václavík, K. Syslová, J. Přech, J. Pecháček, P. Šot, J. Januščák, J. Toman, J. Zápal, M. Kuzma and P. Kačer, "Two Optimized Synthetic Pathways toward a Chiral Precursor of Mivacurium Chloride and Other Skeletal Muscle Relaxants," Tetrahedron: Asymmetry, Vol. 24, No. 1, 2013, pp. 50-55. doi:10.1016/j.tetasy.2012.11.012

[10] M. Kajita, T. Niwa, M. Fujisaki, M. Ueki, K. Niimura, M. Sato, K. Egami, M. Naoi, M. Yoshida and T. Nagatsu, "Detection of 1-Phenyl- $N$-methyl-1,2,3,4-tetrahydroisoquinoline and 1-Phenyl-1,2,3,4-tetrahydroisoquinoline in Human Brain by Gas Chromatography-Tandem Mass Spectrometry," Journal of Chromatography B: Biomedical Sciences and Applications, Vol. 669, No. 2, 1995, pp. 345-351. doi:10.1016/0378-4347(95)00106-S

[11] Y. Makino, Y. Tasaki, S. Ohta and M. Hirobe, "Confirmation of the Enantiomers of 1-Methyl-1,2,3,4-tetrahydroisoquinoline in the Mouse Brain and Foods Applying Gas Chromatography/Mass Spectrometry with Negative Ion Chemical Ionization," Biological Mass Spectrometry, Vol. 19, No. 7, 1990, pp. 415-419. doi:10.1002/bms.1200190706

[12] H. Kažoka, O. Rotkaja and L. Varačeva, "Enantioseparation of 1-Phenyl-1,2,3,4-tetrahydroisoquinoline on Polysaccharide-Based Chiral Stationary Phases," Chromatographia, Vol. 73, No. 1, 2011, pp. 123-129. doi:10.1007/s10337-011-1991-9

[13] A. Lee, H. J. Choi, K. B. Jin and M. H. Hyun, "Liquid Chromatographic Resolution of 1-Aryl-1,2,3,4-Tetrahydroisoquinolines on a Chiral Stationary Phase Based on (+)-(18-Crown-6)-2,3,11,12-Tetracarboxylic Acid," Journal of Chromatography A, Vol. 1218, No. 26, 2011, pp. 4071-4076. doi:10.1016/j.chroma.2011.04.088

[14] H. Inoue, D. Matsubara and Y. Tsuruta, "Simultaneous Analysis of 1,2,3,4-Tetrahydroisoquinolines by High-Performance Liquid Chromatography Using 4-(5,6-Dimethoxy-2-phthalimidinyl)-2-methoxyphenylsulfonyl Chloride as a Fluorescent Labeling Reagent," Journal of Chromatography B, Vol. 867, No. 1, 2008, pp. 32-36. doi:10.1016/j.jchromb.2008.03.002

[15] Y. Song, J. Xu, A. Hamme and Y.-M. Liu, "Capillary Liquid Chromatography-Tandem Mass Spectrometry of Tetrahydroisoquinoline Derived Neurotoxins: A Study on the Blood-Brain Barrier of Rat Brain," Journal of Chromatography A, Vol. 1103, No. 2, 2006, pp. 229-234. doi:10.1016/j.chroma.2005.11.014

[16] M. Cai and Y.-M. Liu, "Quantification of Salsolinol Enantiomers by Stable Isotope Dilution Liquid Chromatography with Tandem Mass Spectrometric Detection," Rapid Communications in Mass Spectrometry, Vol. 22, No. 24, 2008, pp. 4171-4177. doi:10.1002/rcm.3847

[17] N. R. Srinivas, W. C. Shyu and R. H. Barbhaiya, "Gas Chromatographic Determination of Enantiomers as Diastereomers Following Pre-Column Derivatization and Applications to Pharmacokinetic Studies: A Review," Biomedical Chromatography, Vol. 9, No. 1, 1995, pp. 1-9. doi:10.1002/bmc. 1130090102 
[18] E. Gil-Av and D. Nurok, "Resolution of Optical Isomers by Gas Chromatography of Diastereomers," In: J. C. Giddings and R. A. Keller, Eds., Advances in Chromatography, Marcel Dekker, New York, 1974, pp. 99-172.

[19] J. A. Dale, D. L. Dull and H. S. Mosher, " $\alpha$-Methoxy- $\alpha-$ trifluoromethylphenylacetic Acid, a Versatile Reagent for the Determination of Enantiomeric Composition of Alcohols and Amines," Journal of Organic Chemistry, Vol. 34, No. 9, 1969, pp. 2543-2549.

doi:10.1021/jo01261a013

[20] F. Shimoma, H. Kusaka, K. Wada, H. Azami, M. Yasunami, T. Suzuki, H. Hagiwara and M. Ando, "A Novel Synthetic Method of the $( \pm)-(3 \mathrm{a} \alpha, 8 \mathrm{a} \alpha)$-Ethyl $8 \beta$-Hydroxy$6 \beta$-methyl-2-oxooctahydro-2H-cyclohepta[ $[b]$ furan- $3 \alpha$-car boxylate and Its Chemical Transformation to $( \pm)-(3 \mathrm{a} \alpha$, $8 \mathrm{a} \alpha)-3 \alpha, 6 \beta$-Dimethyl-3,3a,4,5,6,8a-hexahydro-2H-cycloh epta[b]furan-2-one, (+)- and (-)-7 $\beta$ - $(2-$ Acetoxy- $1 \alpha-$ methylethyl)- $4 \beta$-methyl-2-cyclohepten- $1 \beta$-ol, and $(+)-$ and (-)-7 $\beta$-(2-Acetoxy-1 $\alpha$-methylethyl)-4 $\beta$-methyl-2-cyclohe pten-1-one. Possible Common Synthetic Intermediates for Pseudoguaianolides, 4,5-Secopseudoguaianolides, Guaianolides, 4,5-Secoguaianolides, and Octalactins," Journal of Organic Chemistry, Vol. 63, No. 4, 1998, pp. 920-929. doi:10.1021/jo971529q
[21] D. A. Allen, A. E. Tomaso Jr., O. P. Priest, D. F. Hindson and J. L. Hurlburt, "Mosher Amides: Determining the Absolute Stereochemistry of Optically-Active Amines," Journal of Chemical Education, Vol. 85, No. 5, 2008, pp. 698-700. doi:10.1021/ed085p698

[22] C. Schotes and A. Mezzetti, "Asymmetric Diels-Alder Reactions of Unsaturated $\beta$-Ketoesters Catalyzed by Chiral Ruthenium PNNP Complexes," Journal of American Chemical Society, Vol. 132, No. 11, 2010, pp. 3652-3653. doi:10.1021/ja910039e

[23] A. M. Gulamhussen, P. Kačer, J. Přech, M. Kuzma and L. Červený, "Highly Efficient Preparation of $(R)$-1-Methyltetrahydroisoquinoline Using Chiral Ru(II)-Catalyst," Reaction Kinetics and Catalysis Letters, Vol. 97, No. 2, 2010, pp. 335-340. doi:10.1007/s11144-009-0036-y

[24] L. Chaoqun and X. Jianliang, "Asymmetric Hydrogenation of Cyclic Imines with an Ionic Cp* Rh(III) Catalyst," Journal of American Chemical Society, Vol. 130, No. 40, 2008, pp. 13208-13209. doi:10.1021/ja8050958

[25] D. J. Ager, A. H. M. de Vries and J. G. de Vries, "Asymmetric Homogeneous Hydrogenations at Scale," Chemical Society Reviews, Vol. 41, 2012, pp. 3340-3380. doi:10.1039/C2CS15312B 